\title{
Defects and dislocations in MgO: atomic scale models of impurity segregation and fast pipe diffusion
}

\author{
FeiwuZhang $^{\mathrm{a}}$, Andrew M Walker ${ }^{\mathrm{b}}$, Kate Wright ${ }^{\mathrm{a}}$ and Julian D Gale ${ }^{\mathrm{a}}$ \\ ${ }^{a}$ Nanochemistry Research Institute, Department of Chemistry, Curtin University of \\ Technology, PO Box U1987, Perth, WA 6845, Australia \\ ${ }^{\mathrm{b}}$ Department of Earth Sciences, University College London, Gower Street, London, \\ WC1E 6BT, UK
}

\section{Graphical contents entry}

Atomic scale simulations show the path taken by magnesium vacancies undergoing rapid pipe diffusion along the core of a $1 / 2<110>\{1 \overline{1} 0\}$ edge dislocation in $\mathrm{MgO}$.

\section{Summary}

Dislocations are known to influence the formation and migration of point defects in crystalline materials. We use a recently developed method for the simulation of the cores of dislocations in ionic materials to study the energy associated with the formation of point defects close to the core of a $1 / 2<110>\{1 \overline{1} 0\}$ edge dislocation in $\mathrm{MgO}$. These are compared then with the energies for the same point defects in otherwise perfect $\mathrm{MgO}$. It is find that all defect species are bound to the dislocation core, with binding energies of between 1.5 and $2.0 \mathrm{eV}$. Vacancies are found to be most stable when they remove undercoordinated ions at the tip of the extra half plane, while the impurities are most stable within the dilatational stress field below the glide plane. By mapping the distribution of energies for point defects around the dislocation line we reveal the coupling between the effective point defect size and the stress field associated with the dislocation. We also examine the energy barrier to diffusion of vacancies along the dislocation line and find that vacancy migration along the dislocation line will be substantially enhanced compared to migration through the dislocation free crystal structure. Activation energies are $0.85-0.92$ of the barrier in the perfect crystal, demonstrating the importance of pipe diffusion along extended defects for low temperature mobility in ionic materials. 


\section{Introduction}

The formation and migration of point defects in ionic materials plays a key enabling role in a wide range of applications and processes ranging from the operation of solid oxide fuel cells and battery materials ${ }^{1}$, to the kinetics of reconstructive phase transitions, equilibrium between phases $^{2}$ and the deformation of the Earth's deep interior ${ }^{3}$. Although atomic scale simulation has been extensively used to probe details of the formation and migration of point defects, studies of how larger-scale defects change this behaviour have been limited, and have tended to concentrate on the effect of grain boundaries and interfaces $^{4}$. In this work we make use of atomic scale simulation methods to show how dislocations can influence the formation and migration of point defects in the prototypical ionic material, $\mathrm{MgO}$.

Dislocations are an important class of one-dimensional extended defect that are best known for their role as the agent of plastic deformation and as enablers of crystal growth ${ }^{5}$. Their orientation and distribution are commonly observed using the transmission electron microscope (TEM), but it is only now becoming possible to understand the structure of the dislocation cores from a combination of new experimental methods ${ }^{6}$ and atomic scale computer simulation ${ }^{7,8}$. However, dislocations are also known to interact with point defects. In the case of metals, it is generally assumed that impurity defects will pin dislocations $^{9}$, leading to a reduction in the rate of dislocation multiplication and hence in creep rate for any given stress. The mechanism for this was first discussed by Cottrell and involves the interaction of the stress field of the dislocation and point defect; a mechanism that can be understood without recourse to the atomic scale ${ }^{10}$. Dislocation cores can also act as fast diffusion pathways that significantly enhance overall diffusion rates. The degree of enhancement is dependent on factors such as dislocation density, orientation and arrangement ${ }^{5}$. This so-called 'pipe diffusion' has been measured in bulk samples, and, remarkably, even for single dislocations ${ }^{11,12}$. Even so, is difficult to explicitly measure pipe diffusion along dislocation cores where the activation energy is generally taken to be of the order of $\sim 0.6$ of that in the bulk lattice ${ }^{13}$.

Atomistic simulation methods have been enormously successful in elucidating point defect behaviour in complex phases, and providing insights into migration mechanisms 
and activation energies ${ }^{14}$ not readily available from experiment. Previous simulations based on 2D geometries, have investigated grain-boundary process ${ }^{15}$. The use of $1 \mathrm{D}$ methods for investigating the structure of dislocations in ionic solids is still in its infancy but is already proving to be a valuable tool ${ }^{8,16}$. For example, simulations by Walker et $\mathrm{al}^{17}$ on siliceous zeolite A (LTA) showed how the introduction of a screw dislocation, observed by $\mathrm{AFM}^{18}$, would lead to blocking of channels normal to the dislocation line. More recently, methods based on density functional theory and the Peierls-Nabarro model have been used to provide detailed structural information on dislocation structures in a wide range of minerals and ceramics including perovskites, $\mathrm{MgSiO}_{4}$ spinel and forsterite $^{19}$. In this study, we investigate the interaction of point defects (vacancies and impurities) with the $1 / 2<110>\{1 \overline{1} 0\}$ edge dislocation in $\mathrm{MgO}$. The approach allows us to calculate binding energies between the point defects and dislocations and activation energies for diffusion along the dislocation core. This work represents such simulation, which should be generally applicable to any ionic material.

\section{Methods}

We study the interactions between dislocation cores and point defects using a new parameterized interatomic potential model and by building on previously developed methods used to study isolated dislocations in crystals ${ }^{20}$. We choose to examine the $1 / 2<110>\{1 \overline{1} 0\}$ edge dislocation in $\mathrm{MgO}$ as this has a comparatively simple structure and has been the subject of several previous studies ${ }^{7}$. The general approach is to construct a model of the isolated dislocation and find a low energy structure of the dislocation core. We can then introduce point defects, in the form of vacancies or impurities, around the dislocation core and compare the energetic cost of forming these defects with the cost of introducing an equivalent defect in an $\mathrm{MgO}$ crystal away from a dislocation line. The final stage of our calculations involves examining the energy barrier to moving the point defects along the dislocation line, and comparing this with diffusion in a region of the crystal away from the dislocation. All calculations were performed with the latest revision of General Utility Lattice Program (GULP) ${ }^{21}$. 
In order to reproduce the cubic rock salt structure and Cauchy violation (i.e. $\boldsymbol{C}_{12} \neq \boldsymbol{C}_{44}$ ) in $\mathrm{MgO}$, it is necessary to use a breathing shell model. The most significant effect of introducing a breathing term is well known ${ }^{22}$; the breathing makes equal contributions to softening $\boldsymbol{C}_{11}$ and $\boldsymbol{C}_{12}$, but has no effect on the value of $\boldsymbol{C}_{44}$. We fit a new set of potentials that reproduces the structure, elastic constants, and high and low frequency dielectric constants under ambient conditions. The breathing shell model potentials describe the interactions between the atomic 'core' with 'shell' or 'breathing shell', where 'shell' denotes a potential that acts on the central position of the shell and 'breathing shell' denotes an interaction that acts on the radius of the shell, which was given an equilibrium radius of $1.2 \AA$. The charge on the $\mathrm{Mg}$ ion is +2 , while the core and shell charges for $\mathrm{O}$ are +0.8 and -2.8 , respectively. The maximum interatomic potential cut-off was set to be $10.0 \AA$. In order to make the energy, including its first and second derivatives remain continuous, a polynomial tapering function was applied to ensure that the potential goes smoothly to zero over the taper range of $1.0 \AA$. The damped and truncated real space summation method of Wolf et al. ${ }^{23}$ was used for the efficient calculation of the Coulomb contribution to the energy. We use a damping parameter, $\beta$, of $0.20 \AA^{-1}$ and a cut-off distance, $\mathrm{R}_{\mathrm{C}}$, of $16.0 \AA$. These parameters resulted in the calculated electrostatic energy of bulk $\mathrm{MgO}$ having a good agreement with that calculated using the Ewald summation.

The $\mathrm{MgO}$ model was used to construct a simulation cell that is periodic in one dimension and with a circular cross section. The outer edge of the model is held fixed in order to reproduce the effect of an extended crystal and isolate the centre of the model from the surrounding vacuum. The edge dislocation is introduced into this simulation cell by applying the elastic displacement field for the dislocation in an infinite continuum to the atomic positions (Figures 1 and 2) using the methodology described by Walker et al. ${ }^{20}$ The modelled dislocation is thus periodic along the dislocation line while perpendicular to the dislocation line the modelled crystal can be considered infinite and non-periodic; i.e. the dislocation is isolated. The simulation cell is divided into two regions. The part of the crystal in the outer region is a long distance from the dislocation line, and therefore experiences a relatively small degree of strain. Thus it can be described as an anisotropic elastic continuum. The atomic positions in the inner region are relaxed to an energy 
minimum, resulting in our initial model of a dislocation that will be used to study the interactions of point defects with dislocations.

Point defects are introduced into the 1D model of the dislocation by removing $\mathrm{Mg}$ or $\mathrm{O}$ ions to create vacancies, or by replacing $\mathrm{Mg}$ with another alkaline earth metal to produce isovalent cation impurities. The atomic positions in the inner region are again relaxed to find an energy minimum representing a point defect close to a dislocation line. We are interested in how the energetic cost of introducing the impurity varies around the dislocation line (the dislocation breaks the translational symmetry of the crystal such that the $\mathrm{Mg}$ and $\mathrm{O}$ sites are no longer equivalent). Thus we performed a search over many different sites for this point defect up to $\sim 10 \AA$ from the origin of the dislocation displacement field. In order to limit point defect - point defect interaction, multiple lattice vectors along the dislocation line are included to increase the thickness $(h)$ of the simulation cell. Details of the simulated edge dislocation model and the convergence tests of energies with respect to the system size are given in the following section.

We introduce two measures of point defect stability. The first is the defect energy, the energy difference between a model containing a point defect and a model without the point defect. This is the energy change associated with moving an ion out of the crystal to form a vacancy, moving an isolated ion into the crystal to form an interstitial or combining both operations to form an impurity. In our model, isolated ions define the reference zero point of the potential energy of the system. The second measure of point defect stability is the binding energy for a point defect to a dislocation. This is just the difference between the defect energy of the point defect close to the dislocation and the point defect in an otherwise perfect crystal (with the energy of a defect free dislocation and perfect crystal appropriately included in the energy cycle). This is a measure of how strongly the dislocation attracts (positive binding energy) or repels (negative binding energy) the point defect.

The final stage of the calculation is to probe the mobility of point defects along the dislocation line. This is achieved by creating two vacancies and an interstitial ion; the interstitial is sequentially moved in small steps between the two vacancies and the geometry relaxed under the constraint that the interstitial does not move in the direction 
parallel to the dislocation line (i.e. it is fixed in this direction but can relax perpendicular to the line). While it is in principle possible to locate the transition state analytically through the use of rational function optimisation, the system size generally precludes the use of the Hessian matrix for the present systems. Plotting a graph of energy against interstitial displacement shows the energy barrier to diffusion by the vacancy mechanism. The difference between the energy minimum and maximum on this pathway is the energy barrier to migration, ignoring the energy cost of creating the point defect.

\section{Results and discussion}

The fitted breathing shell model potential parameters for magnesium oxide are described in Table 1. Table 2 shows the calculated properties for perfect $\mathrm{MgO}$ under ambient conditions with the fitted parameters. The simulation results are in excellent agreement with experimental values for the elastic properties, taken from reference ${ }^{24}$. For the isotropic polycrystalline bulk $(K)$ and shear $(G)$ moduli, the Voigt-Reuss-Hill average values are taken, though the variation between the Voigt and Reuss limits is small.

The energy-minimised structure of the point-defect-free dislocation core is shown in Figure 2, where $\mathrm{Mg}-\mathrm{O}$ distances less than $2.0 \AA$ are emphasised by the inclusion of bonds. A single mirror plane is preserved passing through the dislocation core, while along the dislocation line the structure consists of strings of alternating $\mathrm{Mg}$ and $\mathrm{O}$ ions. The dislocation core contains a string of five coordinate ions at the tip of the extra halfplane of atoms (marked M1 in Figure 2). The $\mathrm{Mg}-\mathrm{O}$ distances around the central M1 ion are shorter than those found in the bulk crystal $(2.11 \AA)$ with M1-O1 being $1.94 \AA$ and the two M1-O4 distances being $1.98 \AA$. All of the out of plane distances are unaltered by the introduction of the dislocation. Octahedra close to the core on the glide plane are strongly deformed and contracted, but deformation above and below the glide plane is less obvious. However, closer examination of the bond distances shows that atomic separations above the glide plane are generally compressed while those below the glide plane are extended (Table 3).

Using this model as a starting point, we constructed a 1D supercell to contain the point defects. Results of convergence testing of the defect energy for a magnesium vacancy 
with the supercell size $(h=n \times a)$ and the inner region radius $\left(\mathrm{R}_{1}\right)$ are shown in Figure 3a and $3 b$, respectively. These results show that good convergence is achieved with a cell thickness of $8.452 \AA$ and an inner radius of $40 \AA$. The radius of the full atomistic cell was taken as $60 \AA$ as this guarantees that the relaxed atoms do not interact with the outer edge of the system. In total there are 10,148 ions in our simulated dislocation model.

The defect energy for vacancies as a function of their position in relation to the dislocation was determined by introducing the vacancy at all unique positions close to the dislocation. Two-dimensional contours of the defect energy of magnesium and oxygen vacancies are plotted in Figure $4 \mathrm{a}$ and $4 \mathrm{~b}$, which have the origin of the dislocation at the centre of the plot. As the vacancies approach the dislocation, their energy varies depending on their location with respect to the dislocation core and the position of the extra half plane of ions. For magnesium, the defect energy of vacancies around the dislocation mirrors the elastic strain field. Above the dislocation line, where the extra half plan of ions exists, $\sigma_{\mathrm{xx}}$ (using the standard dislocation-centric setting for the stress field with the Cartesian x-axis parallel to the Burgers [110] vector, the z-axis along the [001] dislocation line and the $y$-axis perpendicular to the (1 $\overline{1} 0)$ glide plane) and $\sigma_{y y}$ are negative and the strain field is compressional; atoms are squeezed together to make room for the extra half plane effectively increasing the pressure in this region. Below the dislocation line $\sigma_{\mathrm{xx}}$ and $\sigma_{\mathrm{yy}}$ are positive and the strain field is dilatational. Planes of atoms are pulled apart to avoid a discontinuity across the glide plane and the effective pressure is decreased. In the region with a compressional strain field the vacancies become less stable than those in perfect $\mathrm{MgO}$. In contrast, the region with a dilatational strain field favours vacancies in comparison to the perfect crystal. This is in agreement with the previously observed tendency for increasing pressure to decrease the stability of vacancies in $\mathrm{MgO}^{25}$. A similar picture emerges for oxygen vacancies, although the highenergy field above the dislocation line is more extensive.

We also calculate defect energies in a perfect simulation cell of the same dimensions as the cell containing the dislocation. These are given in Table 4 and are in agreement with previous values calculated using interatomic potentials ${ }^{26}$ and experimental measurements ${ }^{12}, 27,28$. The lowest energy positions for the magnesium and oxygen vacancies at the dislocation are found at the tip of the extra half plane of atoms (the M1 
site in Figure 2), removing the under-coordinated ion. Binding energies are $-1.7 \mathrm{eV}$ and $1.5 \mathrm{eV}$ for magnesium and oxygen vacancies, respectively. This suggests that vacancies will strongly partition to dislocations in $\mathrm{MgO}$.

We have also investigated the migration of vacancies through the perfect and dislocated simulation cells in order to determine the change in activation energy for pipe diffusion along the dislocation line in the [001] direction. We examined the energy barrier for defect migration between the lowest energy sites, which are not adjacent in the dislocation structure. The lowest energy adjacent site is in the mirror plane immediately below the dislocation line (from M1 to the site behind O1). We assume that pipe diffusion will consist of a pair of hops between these sites, with the vacancy switching between its ground state and a higher energy metastable site. Two of these hops move the vacancy by one lattice vector along the dislocation line. Elementary hops for vacancy diffusion in perfect $\mathrm{MgO}$ are in the $\langle 110\rangle$ direction and a pair of these can move the dislocation by one lattice vector in the [001] direction. Figures 5a and 5b show the energy profiles for magnesium and oxygen vacancy migration, respectively, with an energy barrier of $1.65 \mathrm{eV}$ for $\mathrm{Mg}$ and $1.92 \mathrm{eV}$ for $\mathrm{O}$. These are both smaller than then equivalent barrier in the perfect bulk crystal, which were found to be $1.92 \mathrm{eV}$ for $\mathrm{Mg}$ and $2.08 \mathrm{eV}$ for $\mathrm{O}$, using the same methodology. Thus dislocations will act as pipes allowing material to be rapidly transported through crystals of $\mathrm{MgO}$. For $\mathrm{Mg}$ the core diffusion activation energy is $\sim 0.85$ of the perfect bulk value while for $\mathrm{O}$ it is $\sim 0.92$ of the perfect bulk value, both values are slightly higher than that proposed for metals ${ }^{13}$. The fastest vacancy migration pathway along the dislocation is shown for magnesium in Figure 6.

Finally, we have explored how the energies of $\mathrm{Ca}$ and $\mathrm{Sr}$ impurities vary as they are brought close to the edge dislocation. Figure 7 shows how the defect energies of the substitution vary in the vicinity of the dislocation core in $\mathrm{MgO}$. The lowest defect energies for both $\mathrm{Ca}$ and $\mathrm{Sr}$ are found three atomic layers below the dislocation core where the stress field is dilatational. The calculated $\mathrm{Ca}$ and $\mathrm{Sr}$ defect energies in the bulk $\mathrm{MgO}$ are $5.53 \mathrm{eV}$ and $9.46 \mathrm{eV}$, respectively. The defect energies are $1.0-2.5 \mathrm{eV}$ lower in the dislocation structure than in the bulk system, while being $0.7-1.0 \mathrm{eV}$ higher in the dense structure above the dislocation core. Our results suggest that the $\mathrm{Ca}$ and $\mathrm{Sr}$ impurities will segregate towards the $\mathrm{MgO}$ edge dislocation core. Previous experiments 
and calculations suggest that $\mathrm{Ca}$ segregates to the $\mathrm{MgO}$ grain boundary and induces a structural transformation. ${ }^{29}$ An edge dislocation can be taken as an extreme end member case of a low angle grain boundary. Due to the size difference for $\mathrm{Sr}$ and $\mathrm{Ca}$ (whose ionic radii differ by about $14 \%$ ), we expect larger outward relaxation for $\mathrm{MgO}+\mathrm{Sr}$ compared to $\mathrm{MgO}+\mathrm{Ca}$. The substitution formation energy contour plots (Figure 7) show a higher and wider contour field in $\mathrm{Sr}$ substitution system than for Ca substitution.

\section{Conclusion}

In all cases, we find that the point defects have a lower energy close to the core of the $1 / 2<110>\{1 \overline{1} 0\}$ edge dislocation than in a perfect crystal of $\mathrm{MgO}$. This stabilisation comes from two origins. The tip of the extra half-plane forming the dislocation consists of under-coordinated ions. These are less strongly bound in the structure resulting in low defect energies for creating vacancies at these sites. In addition to this effect, the stress field around the dislocation results in a region below the glide plane that is held in tension and a compressed region above the glide plane. All the point defects we studied are effectively larger than the atoms they replace ( $\mathrm{Ca}$ and $\mathrm{Sr}$ have a larger ionic radius than $\mathrm{Mg}$, and the primary mode of relaxation around the vacancies is outwards) so the point defects are more stable below the glide plane. The magnitude of the stabilisation, the binding energy, is quite large, amounting to $1.0-2.5 \mathrm{eV}$. This suggests that point defects will be concentrated around dislocations in $\mathrm{MgO}$.

We also find that dislocations have a significant effect on vacancy diffusion along the dislocation line. Activation energies are significantly lower for pipe diffusion along the dislocation line than for diffusion through perfect $\mathrm{MgO}$ implying that pipe diffusion will be more rapid than lattice diffusion. This result is relevant to ongoing uncertainty regarding the diffusion mechanism in $\mathrm{MgO}^{30} \cdot{ }^{18} \mathrm{O}$ tracer diffusion has been measured in ultra-pure polycrystalline samples and these results show anomalously rapid diffusion below about $1600 \mathrm{~K}^{31}$. This low temperature tail suggests that an alternative rapid migration path exists even in the ultra-pure samples, and we suggest that this may be evidence for pipe diffusion. More importantly, pipe diffusion has been directly measured for magnesium in single crystal $\mathrm{MgO}^{12}$. These experiments yield activation energies for 
lattice and pipe diffusion of $2.39 \pm 0.20$ and $1.44 \pm 0.40 \mathrm{eV}$, respectively. This is in close agreement with our calculations, despite the uncertainty associated with the possibility of multiple dislocation characters and Burgers vectors contributing in the experimental result.

Pipe diffusion is expected to be particularly important at low temperature. We can estimate the magnitude of this effect by assuming the pre-exponential factor for lattice and pipe diffusion is the same and using our estimates of the activation energy for both processes (assuming extrinsic diffusion). This probably underestimates the effect, but suggests that at $750 \mathrm{~K}$ the diffusivity associated with pipe diffusion will be $\sim 100$ times higher than the diffusivity associated with lattice diffusion while close the melting temperature (at $2500 \mathrm{~K}$ ) the enhancement is reduced to a factor of four. As well as the impact on functional materials, this can also help elucidate the processes leading to low temperature geochemical effects, such as chemical exchange observed in deformed zircons $^{32}$. The method of studying pipe diffusion described here should be generally applicable to any ionic material and might lay the foundation for extending the approach to more complex materials of technological interest.

\section{Acknowledgements}

FZ, KW and JDG would like to thank the Australian Research Council for support under the Discovery Program (DP0878453), and the NCI and iVEC for computational resources. AMW acknowledges the support of the NERC through a Postdoctoral Research Fellowship (NE/E012922/2).

\section{References}

1. B. Peng and J. Chen, Coord. Chem. Rev., 2009, 253, 2805-2813.

2. D. G. Vlachos, L. D. Schmidt and R. Aris, Surf. Sci., 1991, 249, 248-264.

3. C. G. Sammis, Progress in Materials Science, 2001, 46, 231-247.

4. D. J. Harris, M. A. Khan and S. C. Parker, Phys. Chem. Miner., 1999, 27, 133137; D. J. Harris, G. W. Watson and S. C. Parker, Phys. Rev. B, 1997, 56, $11477-$ 11484; D. J. Harris, G. W. Watson and S. C. Parker, Phys. Rev. B, 2001, 64, 
134101; D. P. Dobson, M. Alfredson, C. Holzapfel and J. P. Brodholt, European Journal of Mineralogy, 2007, 19, 617-622.

5. D. Hull and D. J. Bacon, Introduction to dislocations, Butterworth Heinemann, Oxford, 2001.

6. M. Heggen, L. Houben and M. Feuerbacher, Nat. Mater., 2010, 9, 332 - 336 ; C. L. Jia, A. Thust and K. Urban, Physical review letters, 2005, 95, 225506.

7. M. P. Puls, Philos. Mag. A-Phys. Condens. Matter Struct. Defect Mech. Prop., 1980, 41, 353-368; M. P. Puls and M. J. Norgett, J. Appl. Phys., 1976, 47, 466477; C. H. Woo and M. P. Puls, Philos. Mag., 1977, 35, 1641-1652; C. H. Woo and M. P. Puls, Philos. Mag., 1977, 35, 727-756; P. Carrez, D. Ferré and P. Cordier, Model. Simul. Mater. Sci. Eng., 2009, 17, 11.

8. G. W. Watson, E. T. Kelsey and S. C. Parker, Philos. Mag. A-Phys. Condens. Matter Struct. Defect Mech. Prop., 1999, 79, 527-536; J. Marian, W. Cai and V. V. Bulatov, Nat. Mater., 2004, 3, 158 - 163.

9. N. M. Vlasov and V. A. Zaznoba, Tech. Phys., 2001, 46, 51-54.

10. A. H. Cottrell and B. A. Bilby, Proceedings of the Physical Society. Section A, 1949, 62, 49-62.

11. M. Legros, G. Dehm, E. Arzt and T. J. Balk, Science, 2008, 319, 1646-1649; R. J. Gaboriaud, Journal of Physics D-Applied Physics, 2009, 42; J. Narayan and J. Washburn, Philos. Mag., 1972, 26, 1179-\&.

12. I. Sakaguchi, H. Yurimoto and S. Sueno, Solid State Commun., 1992, 84, 889893.

13. W. Cannon and T. Langdon, Journal of materials science, 1983, 18, 1-50.

14. A. M. Walker, K. Wright and B. Slater, Phys. Chem. Miner., 2003, 30, 536-545; G. R. Gardiner and M. S. Islam, Chemistry of Materials, 2009, 22, 1242-1248; C. A. J. Fisher, V. M. H. Prieto and M. S. Islam, Chemistry of Materials, 2008, 20, 5907-5915.

15. J. H. Harding and D. J. Harris, Phys. Rev. B, 2001, 63.

16. A. M. Walker, Phys. Chem. Miner., 2010, 37, 301-310; G. W. Watson, P. M. Oliver and S. C. Parker, Surf. Sci., 2001, 474, L185-L190.

17. A. M. Walker, B. Slater, J. D. Gale and K. Wright, Nat. Mater., 2004, 3, 715-720.

18. S. Dumrul, S. Bazzana, J. Warzywoda, R. R. Biederman and A. Sacco, Microporous Mesoporous Mat., 2002, 54, 79-88.

19. P. Carrez, A. M. Walker, A. Metsue and P. Cordier, Philos. Mag., 2008, 88, 24772485; D. Ferré, P. Carrez and P. Cordier, Phys. Chem. Miner., 2009, 36, 233-239; P. Carrez, P. Cordier, D. Mainprice and A. Tommasi, European Journal of Mineralogy, 2006, 18, 149-160; P. Carrez, D. Ferré and P. Cordier, Philos. Mag., 2007, 87, 3229-3247; P. Carrez, D. Ferré and P. Cordier, Nature, 2007, 446, 6870; J. Durinck, P. Carrez and P. Cordier, European Journal of Mineralogy, 2007, 19, 631-639; D. Ferré, P. Carrez and P. Cordier, Physics of the Earth and Planetary Interiors, 2007, 163, 283-291; D. Ferré, P. Carrez and P. Cordier, Phys. Rev. B, 2008, 77, 014106; D. Ferré, P. Cordier and P. Carrez, American Mineralogist, 2009, 94, 135-142.

20. A. M. Walker, J. D. Gale, B. Slater and K. Wright, Phys Chem Chem Phys, 2005, 7, 3227-3234; A. M. Walker, J. D. Gale, B. Slater and K. Wright, Phys Chem Chem Phys, 2005, 7, 3235-3242. 
21. J. D. Gale, Journal of the Chemical Society-Faraday Transactions, 1997, 93, 629637; J. D. Gale and A. L. Rohl, Molecular Simulation, 2003, 29, 291-341.

22. M. J. Sangster, Journal of Physics and Chemistry of Solids, 1973, 34, 355-363.

23. D. Wolf, P. Keblinski, S. R. Phillpot and J. Eggebrecht, Journal of Chemical Physics, 1999, 110, 8254-8282.

24. C. S. Zha, H. K. Mao and R. J. Hemley, P Natl Acad Sci USA, 2000, 97, 1349413499.

25. B. B. Karki and G. Khanduja, American Mineralogist, 2006, 91, 511-516.

26. C. R. A. Catlow, I. D. Faux and M. J. Norgett, J Phys C Solid State, 1976, 9, 419429.

27. S. Shirasak and M. Hama, Chem. Phys. Lett., 1973, 20, 361-365.

28. D. R. Sempolinski and W. D. Kingery, J. Am. Ceram. Soc., 1980, 63, 664-669.

29. Y. Yan, M. F. Chisholm, G. Duscher, A. Maiti, S. J. Pennycook and S. T. Pantelides, Physical review letters, 1998, 81, 3675-3678.

30. M. W. Ammann, J. P. Brodholt and D. P. Dobson, Reviews in Mineralogy and Geochemistry, 2010, 71, 201-224.

31. M. H. Yang and C. P. Flynn, Physical review letters, 1994, 73, 1809-1812; M. H. Yang and C. P. Flynn, J. Phys.-Condes. Matter, 1996, 8, L279-L283.

32. S. M. Reddy, N. E. Timms, P. Trimby, P. D. Kinny, C. Buchan and K. Blake, Geology, 2006, 34, 257-260. 


\section{Tables}

Table 1. Fitted breathing shell model potentials for magnesium oxide and $\mathrm{Ca}, \mathrm{Sr}$ impurities. Here core, shel and bshel indicate that the potential acts on the core, shell position or radius of the shell, respectively.

\begin{tabular}{|c|c|c|c|c|c|c|c|}
\hline $\begin{array}{c}\text { Species } \\
1\end{array}$ & $\begin{array}{c}\text { Species } \\
2\end{array}$ & Potential & $A(\mathrm{eV})$ & $\rho(\AA)$ & $\begin{array}{c}C_{6} \\
\left(\mathrm{eV} \AA^{6}\right)\end{array}$ & $k_{\mathrm{CS}}\left(\mathrm{eV} \AA^{-2}\right)$ & $k_{\mathrm{BSM}}\left(\mathrm{eV}^{-2}\right)$ \\
\hline Mg core & O bshel & Buckingham & 30.13372 & 0.30404 & 0.0 & - & - \\
\hline Ca core & O bshel & Buckingham & 45.04151 & 0.32860 & 0.0 & & \\
\hline Sr core & O bshel & Buckingham & 52.22144 & 0.35050 & 0.0 & & \\
\hline O shel & O shel & Buckingham & 0.0 & 0.3 & 36.00 & - & - \\
\hline O core & O shel & Spring & - & - & - & 43.797310 & - \\
\hline O bshel & O bshel & BSM & - & - & - & - & 318.801 \\
\hline
\end{tabular}

Table 2. Calculated and experimental properties for magnesium oxide under ambient conditions. Calculations are static lattice minimizations at this study.

\begin{tabular}{|c|c|c|}
\hline Properties & Experiment & This model \\
\hline$\alpha(\AA)$ & 4.212 & 4.226 \\
\hline$C_{11}[G P a]$ & 297.0 & 297.8 \\
\hline$C_{12}[G P a]$ & 95.2 & 94.9 \\
\hline$C_{44}[G P a]$ & 155.7 & 158.9 \\
\hline$\varepsilon^{0}$ & 9.86 & 9.78 \\
\hline$\varepsilon^{\infty}$ & 2.96 & 3.03 \\
\hline $\mathrm{K}[G P a]$ & 162.5 & 162.5 \\
\hline $\mathrm{G}[G P a]$ & 130.4 & 132.7 \\
\hline $\mathrm{V}_{\mathrm{s}}[\mathrm{km} / \mathrm{s}]$ & 6.06 & 6.11 \\
\hline $\mathrm{V}_{\mathrm{p}}[\mathrm{km} / \mathrm{s}]$ & 9.68 & 9.78 \\
\hline$\sigma$ & 0.18 & 0.179 \\
\hline
\end{tabular}


Table 3.Average $\mathrm{Mg}$ - $\mathrm{O}$ distances for selected sites around the dislocation core. Note that all $\mathrm{Mg}$ and $\mathrm{O}$ sites are inverted in adjacent layers along the dislocation line and the out of plane distances are excluded from the average. In cases where one distance is anomalously long (where it is unclear if the site should be considered 5 or 6 coordinate) we present both options.

\begin{tabular}{llc}
\hline Central site & Adjacent sites & Average Mg-O distance $(\AA)$ \\
\hline M1 & O1, O4, O4 & 1.97 \\
M2 & O4, O4, O5, O5 & 2.06 \\
M5 & O1, O2, O6, O7 & 2.21 \\
M6 & O4, O7, O8 & 1.97 \\
M6 & O1, O4, O7, O8 & 2.32 \\
M7 & O4, O5, O8, O9 & 2.06 \\
M9 & O7, O8, O10, O11 & 2.19 \\
O1 & M1, M5, M5 & 2.06 \\
O1 & M1, M5, M5, M6, M6 & 2.56 \\
O2 & M4, M4, M5, M5 & 2.20 \\
O4 & M1, M2, M6, M7 & 2.02 \\
O7 & M5, M6, M8, M9 & 2.21 \\
O8 & M6, M7, M9, M10 & 2.03 \\
Bulk MgO & & 2.11 \\
\hline
\end{tabular}


Table 4. Calculated and experimental data for the energies $(\mathrm{eV})$ of defects formation and diffusion in $\mathrm{MgO}$ bulk system.

\begin{tabular}{|c|c|c|c|c|}
\hline & \multicolumn{2}{|c|}{ Vacancy } & \multicolumn{2}{|c|}{$\begin{array}{c}\text { Activation } \\
\text { energy of diffusion }\end{array}$} \\
\hline & $V_{M g}^{\prime \prime}$ & $V_{o}^{*}$ & $\mathrm{Mg}$ & $\mathrm{O}$ \\
\hline Calculated (This study) & 23.7 & 23.9 & 1.92 & 2.08 \\
\hline Calculated (Catlow et al. ${ }^{26}$ ) & 23.74 & 24.63 & 1.9 & 2.0 \\
\hline Experimental (Sakaguchi et al. ${ }^{12}$ ) & - & - & $2.4 \pm 0.2$ & - \\
\hline Experimental (Shirasaki and Hama ${ }^{27}$ ) & - & - & - & 2.42 \\
\hline Experimental (Sempolinski and Kingery ${ }^{28}$ ) & - & - & 2.28 & - \\
\hline
\end{tabular}




\section{Figure Captions}

Figure 1. Schematic representation of the simulation cell used in the present work. In this study, we set up the $1 \mathrm{D}$ edge dislocation cell with $\mathrm{h}=2 *_{\mathrm{a}}=8.452 \AA, \mathrm{R}_{1}=40 \AA$ and $\mathrm{R}_{2}=$ $60 \AA$.

Figure 2. The edge dislocation structure of $\mathrm{MgO}$. The dislocation line [001] is perpendicular to the image plane. The Burgers vector of this dislocation is $1 / 2<1 \overline{1} 0>$. 11 $\mathrm{Mg}$ and $\mathrm{O}$ atoms are marked around the dislocation core for describe in Table 3.

Figure 3. (a) Convergence of the Defect energy of Mg vacancy $\left(V_{M g}^{\prime \prime}\right)$ with the thickness of the simulation cell size, $n$, in $\mathrm{MgO}$ bulk and dislocation systems to the left y-axis scale, Open circle is the $V_{M g}^{\prime \prime}$ defect energy difference between bulk and dislocation system ( $\Delta E=E_{\text {Bulk }}-E_{\text {Dislcation }}$ ) to the right y-axis scale. The radius of the inner region is $40 \AA$ and the radius of the outer region is $60 \AA$. (b) Dislocation formation energy as a function of radius of the inner region. The radius of the outer region is $60 \AA$ and the thickness of the simulation cell is $8.452 \AA$.

Figure 4. Contour plots of (a) magnesium and (b) oxygen vacancy defect energies in the vicinity of the edge dislocation. The dislocation line is normal to the page and the dots represent projections of the atoms.

Figure 5. Energy profiles for (a) the magnesium vacancy and (b) the oxygen vacancy diffusion in both dislocation and bulk systems. The migration activation energiesare 1.65 $\mathrm{eV}$ for magnesium and $1.92 \mathrm{eV}$ for oxygen in the dislocation core and $1.92 \mathrm{eV}$ for magnesium and $2.08 \mathrm{eV}$ for oxygen in the bulk $\mathrm{MgO}$. 
Figure 6. The magnesium diffusion path along the dislocation core in $\mathrm{MgO}$.

Figure 7. Contour plots of (a) $\mathrm{Ca}$ and (b) Sr substitution formation energies in the vicinity of the edge dislocation in $\mathrm{MgO}$. The dislocation line is normal to the page and the dots represent projections of the atoms. 
Figure 1

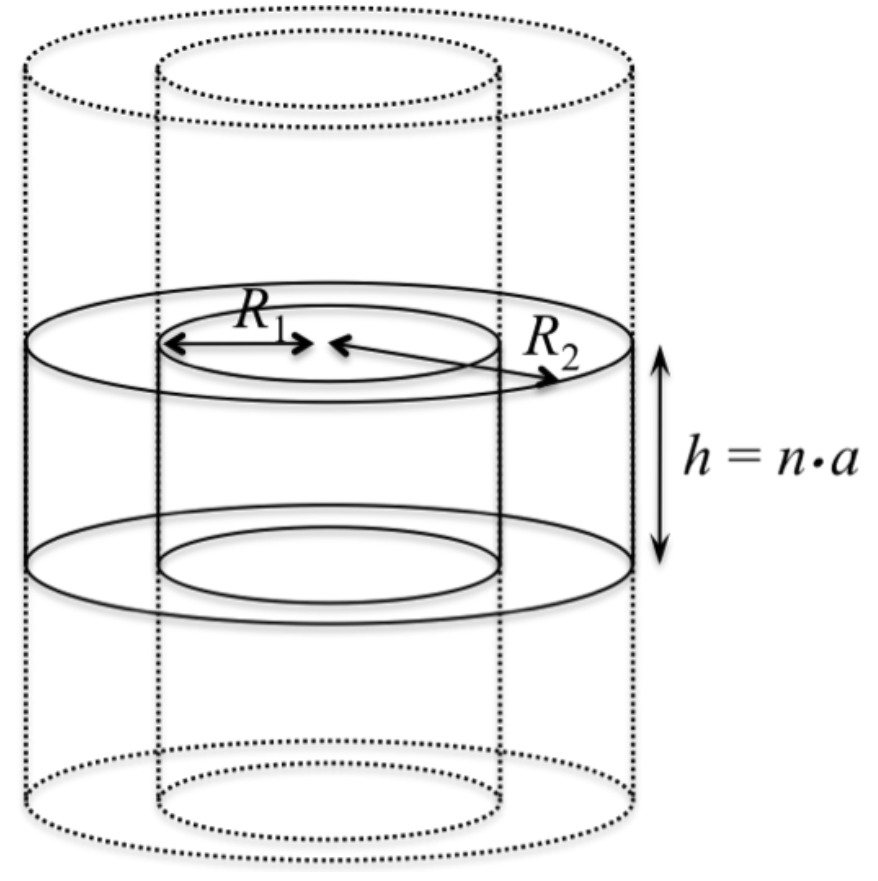


Figure 2

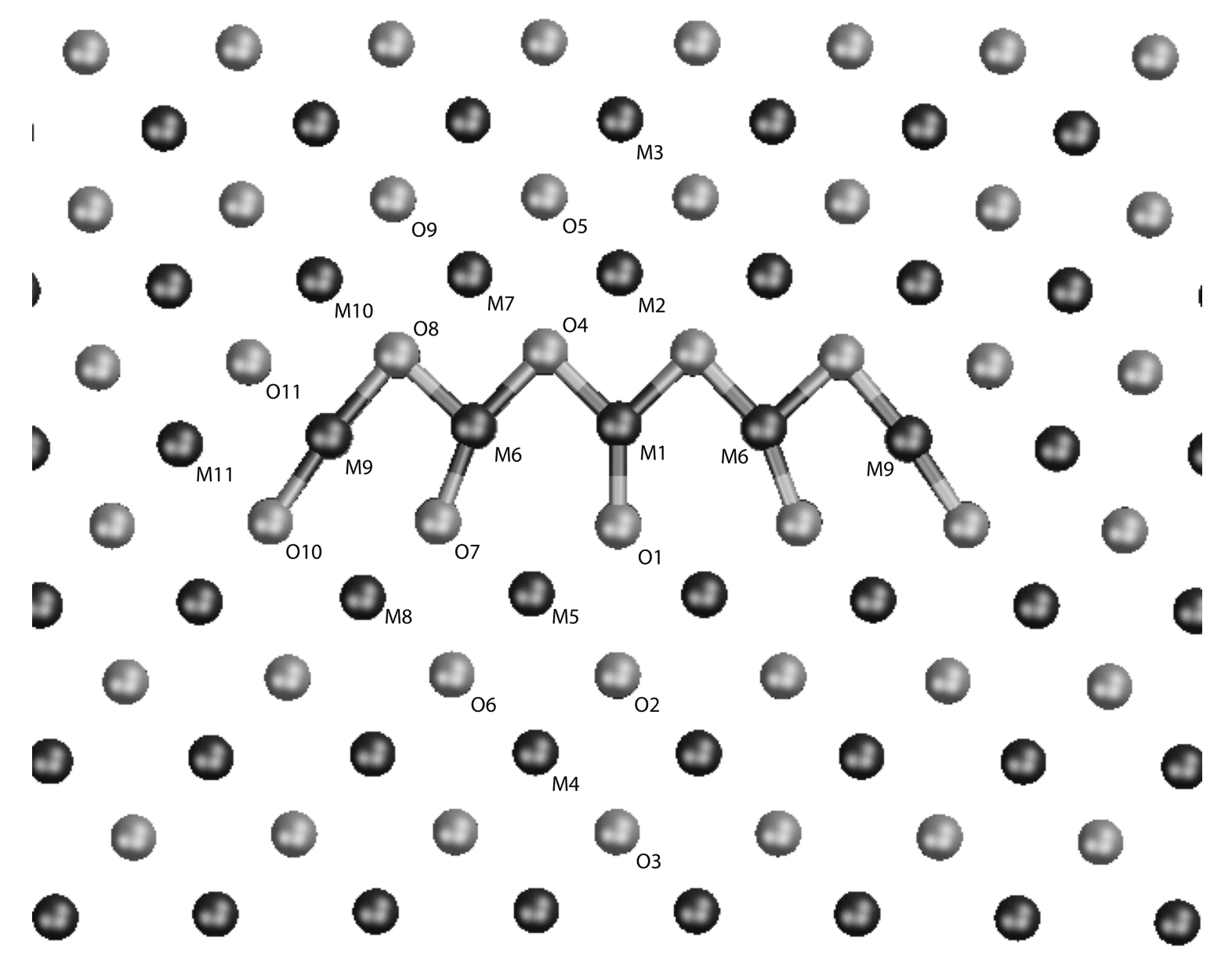


Figure 3
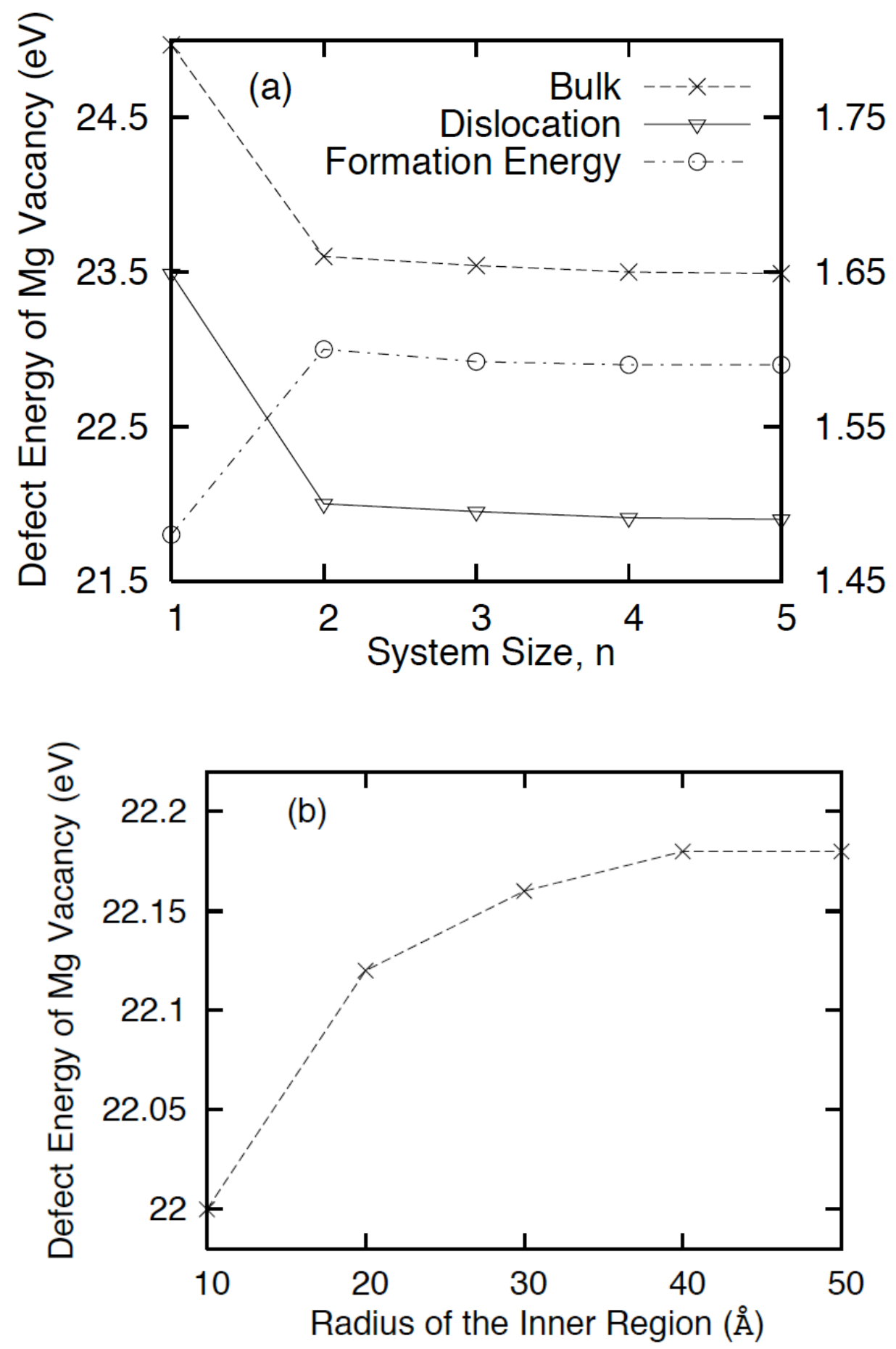
Figure 4

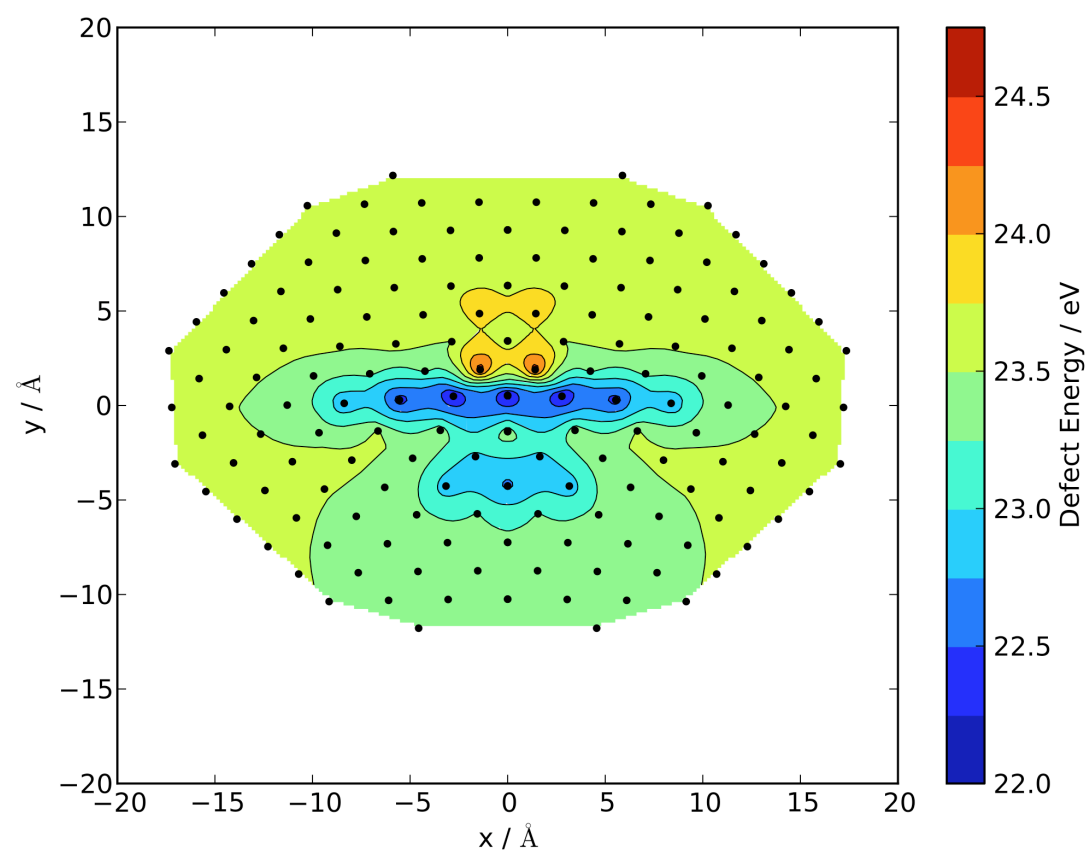

(a)

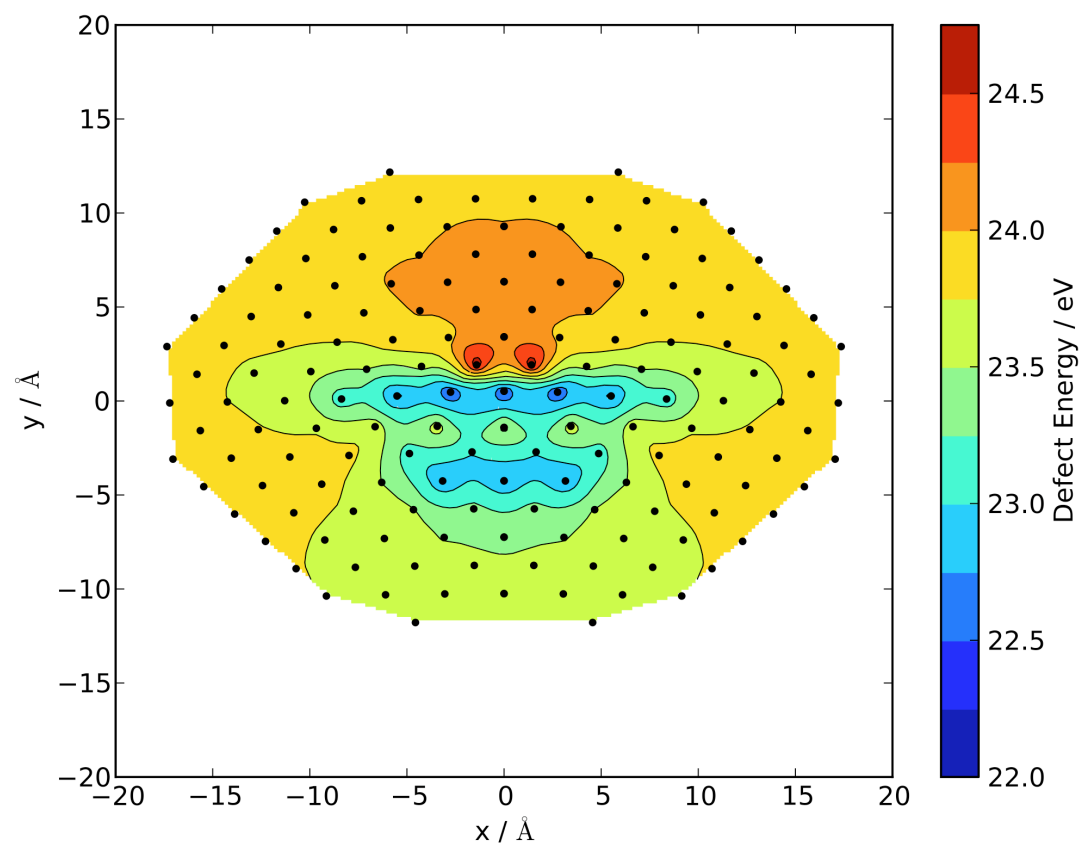

(b). 
Figure 5
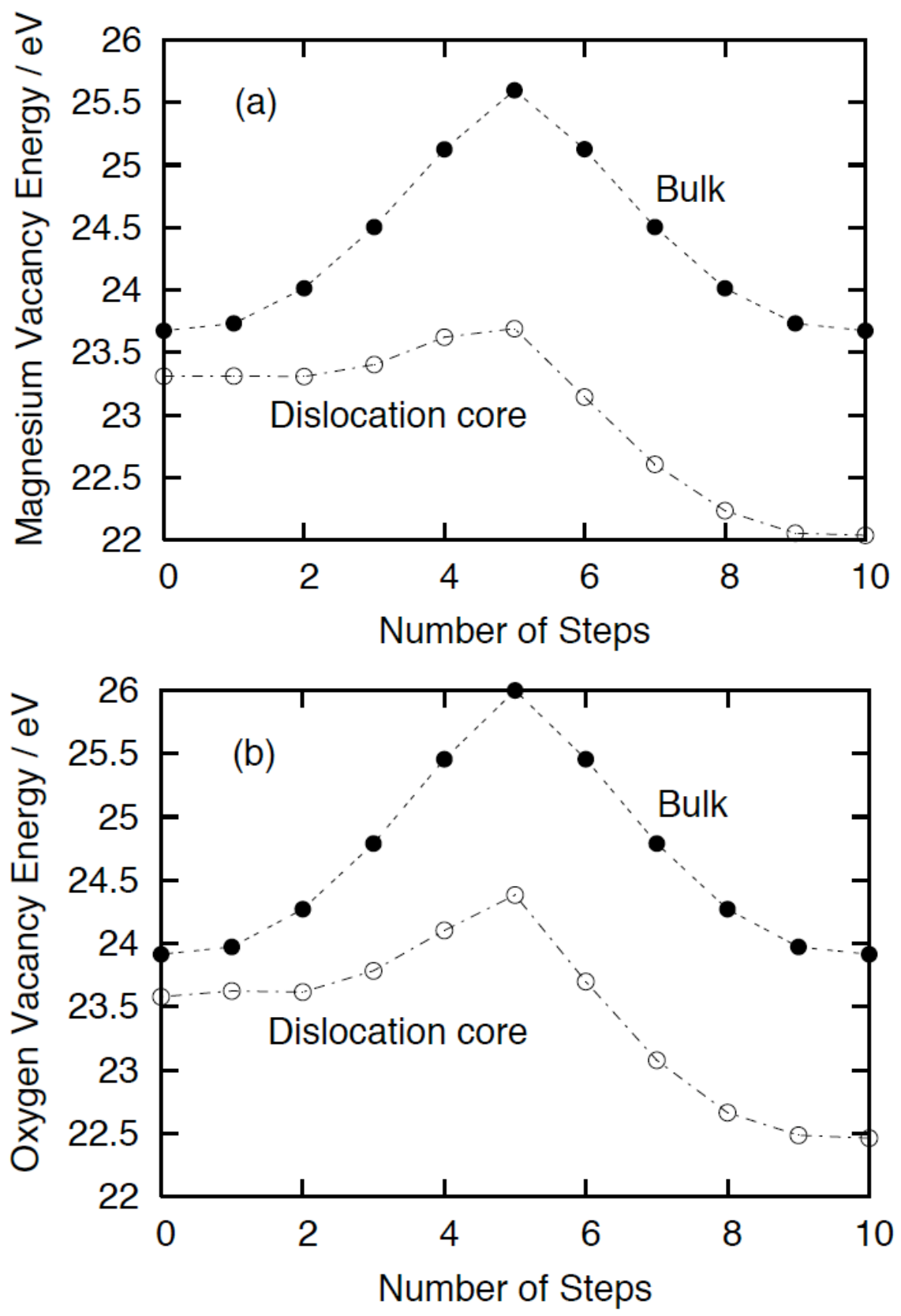
Figure 6

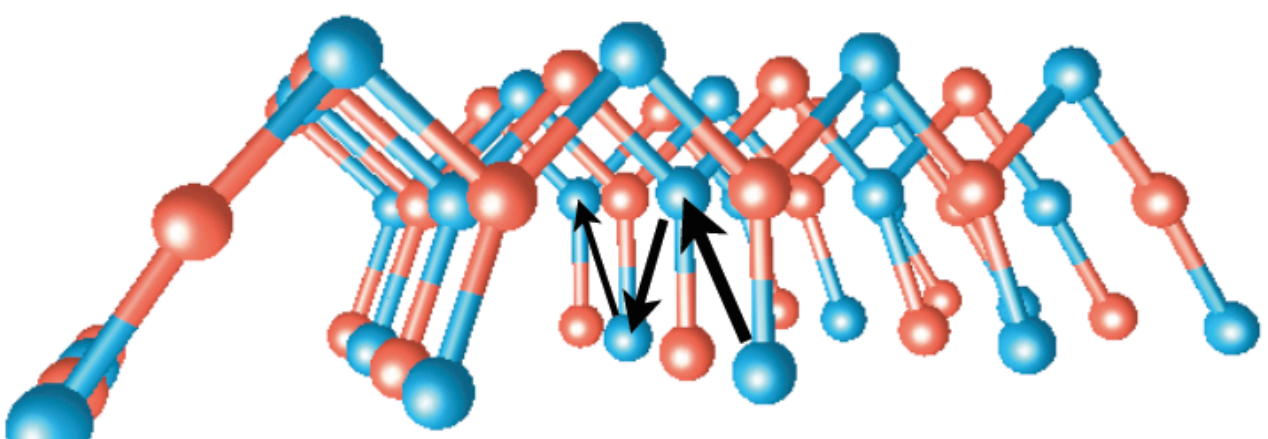

Fast diffusion path 
Figure 7

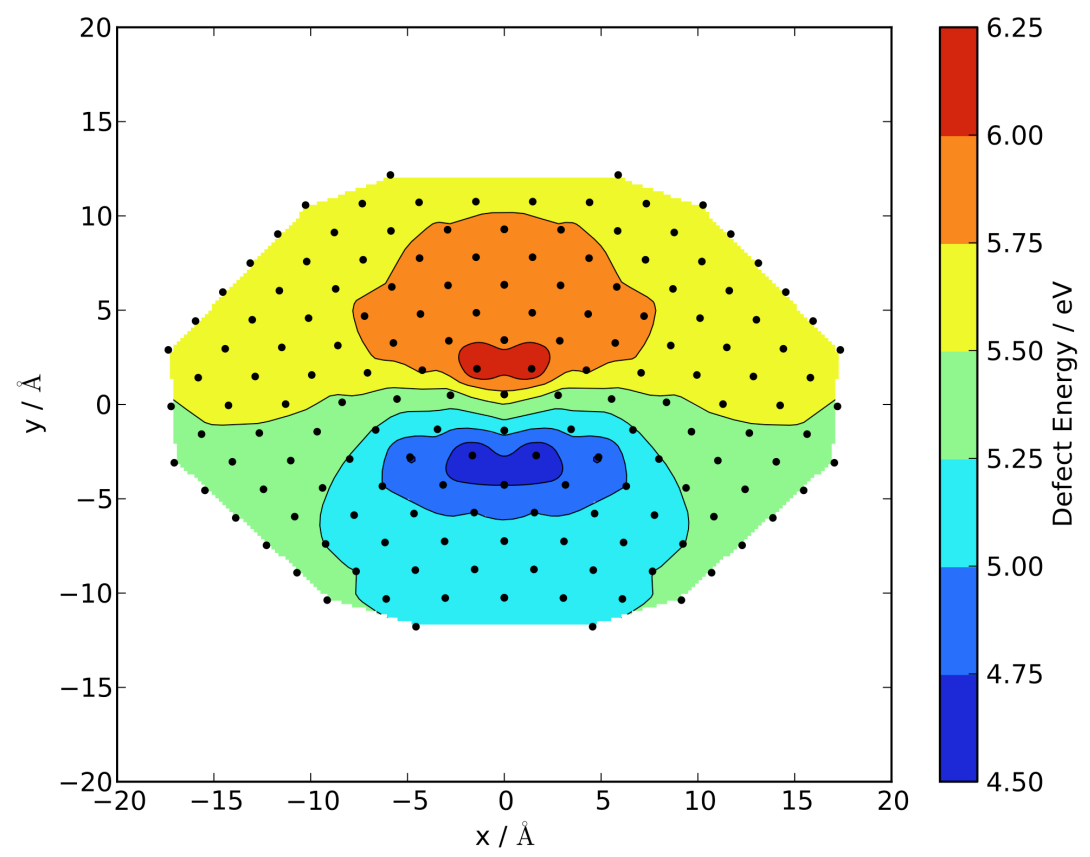

(a)

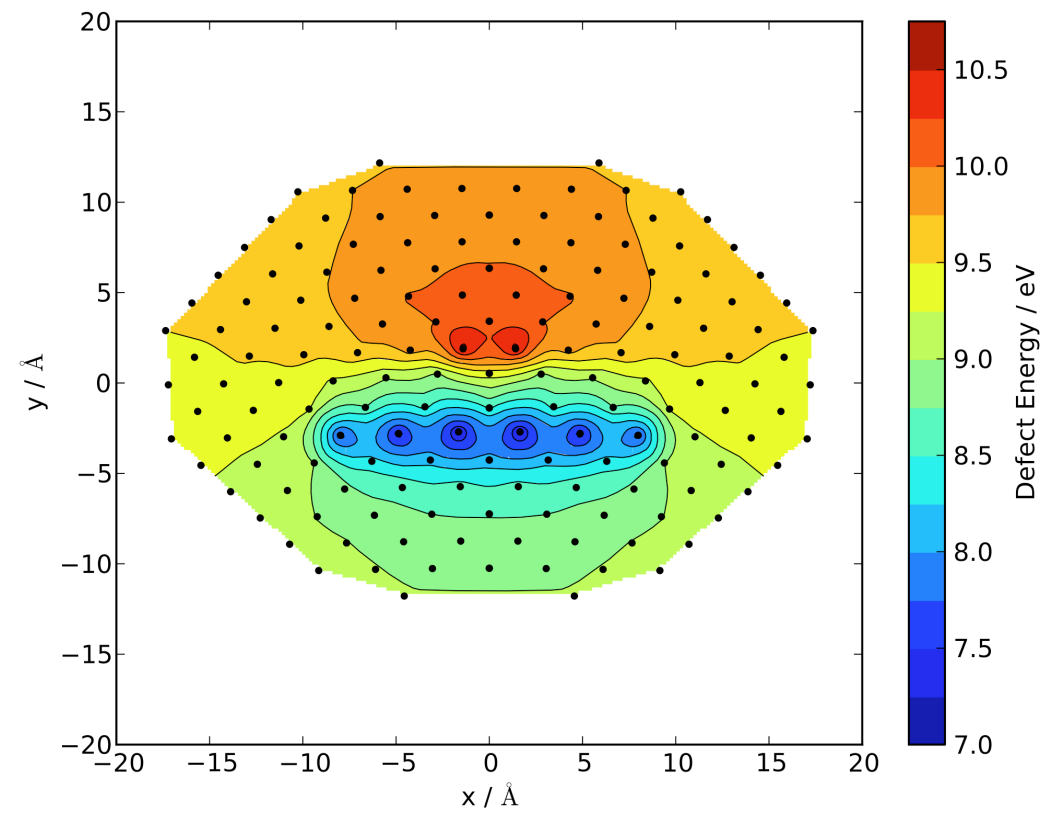

(b) 